\title{
The Poncelet annuli
}

\author{
Waldemar Cieślak
}

Received: 16 November 2012 / Accepted: 25 February 2013 / Published online: 29 March 2013

(C) The Author(s) 2013. This article is published with open access at Springerlink.com

\begin{abstract}
The sufficient and necessary condition for a circular annulus to have the property of Poncelet porism is given. Moreover, the family of annuli with a property of the Poncelet porism and some family of periodic functions are considered. The close relations between these families are given.
\end{abstract}

Keywords Porism · Annulus

\section{Mathematics Subject Classification (1991) 53A04}

\section{Introduction}

A polygon is called circuminscribed in an annulus if it is simultaneously inscribed in the outer curve and circumscribed on the inner curve. Poncelet in Bos et al. (1987) proved a theorem called Poncelet's closure theorem:

If there exists one circuminscribed n-gon in a fixed annulus $C D(C$ and $D$ are smooth conics), then any point of the outer curve is the vertex of some circuminscribed n-gon.

The paper Bos et al. (1987) very precisely describes Poncelet's porism. The literature on the porism is given in Bos et al. (1987) and Weisstein and Poncelet's Porism (2008), recent papers on this subject are given by Radić (2009, 2010). A family of annuli bounded by ovals were considered in e.g. Cieślak and Szczygielska (2008), Mozgawa (2008), Cieślak et al. (2013). We introduce the following definition:

An annulus with a property of the Poncelet porism will be called a Poncelet annulus.

W. Cieślak $(\bowtie)$

Katedra Matematyki Stosowanej, Politechnika Lubelska,

ul. Nadbystrzycka 40, 20-618 Lublin, Poland

e-mail: izacieslak@wp.pl 
Mozgawa (2008) proved that for a given oval $C$ there exists ovals $C_{i n}$ and $C_{\text {out }}$, inside and outside of $C$, such that the annuli $C C_{i n}$ and $C C_{\text {out }}$ are Poncelet annuli (for almost all natural numbers). In Cieślak and Szczygielska (2008) it is proved that each oval and a natural number $n \geq 3$ generate a Poncelet annulus. The papers Cieślak and Szczygielska (2008) and Mozgawa (2008) show that the family of all Poncelet annuli is very wide. In this paper the assumption of convexity of the outer curve in the annulus is not important.

\section{Families $\Phi_{L, n}$ and $\mathcal{B}_{L}$}

In this section we generalize some results of Cieślak (1986). Let us fix a positive number $L$ and a natural number $n \geq 3$.

We denote by $\Phi_{L, n}$ a family of all $C^{1}$-functions $\varphi: \mathbb{R} \rightarrow \mathbb{R}$ satisfying the following conditions

$$
\begin{gathered}
0<\varphi(0)<L, \\
\varphi^{\prime}(t)>0, \\
\varphi^{[n]}(t)=t+L
\end{gathered}
$$

for $t \in \mathbb{R}$, where

$$
\varphi^{[1]}=\varphi, \quad \varphi^{[k]}=\varphi \circ \varphi^{[k-1]}
$$

for natural $k \geq 2$.

We note that $\varphi(t)+L=\varphi^{[n]}(\varphi(t))=\varphi\left(\varphi^{[n]}(t)\right)=\varphi(t+L)$, i.e.

$$
\varphi(t+L)=\varphi(t)+L \quad \text { for all } \quad t \in \mathbb{R}
$$

The inverse function $\varphi^{-1}$ to $\varphi$ is given by the formula

$$
\varphi^{-1}(t)=\varphi^{[n-1]}(t)-L \quad \text { for all } \quad t \in \mathbb{R}
$$

For example the function $\varphi(t)=t+\frac{1}{n} L$ for $t \in \mathbb{R}$ is an element of $\Phi_{L, n}$.

We denote by $\mathcal{B}_{L}$ a family of all continuous functions $b: \mathbb{R} \rightarrow \mathbb{R}$ satisfying the following conditions:

$$
\begin{gathered}
b(t)>0, \\
b(t+L)=b(t)
\end{gathered}
$$

for $t \in \mathbb{R}$.

We associate with each function $b \in \mathcal{B}_{L}$ two functions $B: \mathbb{R} \rightarrow \mathbb{R}$ and $\beta: \mathbb{R} \rightarrow \mathbb{R}$ given by the formulas 


$$
\begin{gathered}
B(t)=\int_{0}^{t} b(u) d u, \\
\beta(t)=B\left(t+\frac{L}{2}\right)-B(t) .
\end{gathered}
$$

We note that

$\beta(t+L)=B\left(t+L+\frac{L}{2}\right)-B(t+L)=B(L)+B\left(t+\frac{L}{2}\right)-B(t)-B(L)=\beta(t)$ i.e.

$$
\beta(t+L)=\beta(t) \text { for } t \in \mathbb{R}
$$

and the function $\beta$ reaches its minimum. Let

$$
\beta_{\min }=\min _{t \in \mathbb{R}} \beta(t)
$$

Now, we give two relations between the families $\Phi_{L, n}$ and $\mathcal{B}_{L}$. By simple verification we have

Lemma 2.1 Let $b \in \mathcal{B}_{L}$. A function $\varphi: \mathbb{R} \rightarrow \mathbb{R}$ defined by the formula

$$
\varphi(t)=B^{-1}\left(B(t)+\frac{1}{n} B(L)\right)
$$

belongs to $\Phi_{L, n}$ and it satisfies the differential eqnarray

$$
\varphi^{\prime}=\frac{b}{b \circ \varphi}
$$

with the initial condition

$$
\varphi(0)=B^{-1}\left(\frac{1}{n} B(L)\right)
$$

The above lemma is a generalization of the Lemma from Cieślak (1986). In view of the conditions (2.2) and (2.5) we have immediately the following result:

Lemma 2.2 If $\psi \in \Phi_{L, n}$ then a function $b: \mathbb{R} \rightarrow \mathbb{R}$ defined by the formula

$$
b=\left(\psi^{[n-1]}\right)^{\prime}+\left(\psi^{[n-2]}\right)^{\prime}+\cdots+\psi^{\prime}+1
$$

belongs to $\mathcal{B}_{L}, \psi^{\prime}=\frac{b}{b \circ \psi}$ and $B(L)=n L$. 
Lemma 2.3 If $\varphi \in \Phi_{L, n}$ then

$$
\varphi(t)-t>0 \quad \text { for all } t \in \mathbb{R} \text {. }
$$

Proof We assume that

$$
\varphi\left(t_{0}\right) \leq t_{0} \quad \text { for some } \quad t_{0} \in \mathbb{R}
$$

The function $\varphi$ is strictly increasing so we have

$$
\varphi^{[2]}\left(t_{0}\right) \leq \varphi\left(t_{0}\right)
$$

The inequalities (2.18) and (2.19) imply that $\varphi^{[2]}\left(t_{0}\right) \leq t_{0}$. Repeating this procedure we get $\varphi^{[n]}\left(t_{0}\right) \leq t_{0}$, i.e. $t_{0}+L \leq t_{0}$. The obtained contradiction proves the inequality (2.17).

\section{A family $\mathcal{B}_{L, n}$}

Let us fix a natural number $n \geq 3$. We define a subfamily $\mathcal{B}_{L, n}$ of $\mathcal{B}_{L}$ as follows: a function $b \in \mathcal{B}_{L}$ belongs to $\mathcal{B}_{L, n}$ if it satisfies the following condition

$$
\beta_{\min }>\frac{1}{n} B(L)
$$

where $B$ and $\beta$ are defined by (2.9) and (2.10), respectively.

Example 3.1 We consider the family $\mathcal{B}_{2 \pi}$. The function $b(t)=\sin t+r$ for $t \in \mathbb{R}$ belongs to $\mathcal{B}_{2 \pi}$ for $r>1$. We have $B(t)=1-\cos t+r t$ and $\beta(t)=B(t+\pi)-B(t)=$ $2 \cos t+r \pi$. Hence we obtain $\min _{t \in \mathbb{R}} \beta(t)=\beta(\pi)=r \pi-2$ and $B(2 \pi)=2 \pi r$. The inequalityeqref2.1 for $n=3$ implies $r>\frac{6}{\pi}$. The function $b$ belongs to $\mathcal{B}_{2 \pi, 3}$ if $r \in\left(\frac{6}{\pi},+\infty\right)$.

Now, we give some properties of the family $\mathcal{B}_{L, n}$.

Lemma 3.1 Let $b \in \mathcal{B}_{L}$ and $b \notin \mathcal{B}_{L, n}$. Then a function $b_{c}: \mathbb{R} \rightarrow \mathbb{R}$ defined by the formula

$$
b_{c}(t)=b(t)+c
$$

belongs to $\mathcal{B}_{L, n}$ if and only if

$$
c>\frac{2 n}{L(n-2)}\left(\frac{1}{n} B(L)-\beta_{\min }\right) .
$$

Proof We have immediately that $B_{c}(t)=B(t)+c t$ and $\beta_{c}(t)=\beta(t)+\frac{1}{2} L c$. Thus the minimum of $\beta_{c}$ is given by the formula

$$
\beta_{c, \min }=\beta_{\min }+\frac{1}{2} L c .
$$


We note that $B_{c}(L)=B(L)+L c$ and the inequality (3.1) for the function $\beta_{c}$ has the form

$$
\beta_{\min }+\frac{1}{2} L c>\frac{1}{n} B(L)+\frac{1}{n} L c .
$$

Hence we obtain (3.3).

Lemma 3.2 Let $b \in \mathcal{B}_{L}$. The function $\varphi$ given by (2.13) satisfies the inequality

$$
\varphi(t)-t<\frac{1}{2} L \text { for } t \in \mathbb{R}
$$

if and only if $b \in \mathcal{B}_{L, n}$.

Proof The inequality (3.4) can be rewritten in the following equivalent forms

$$
\begin{gathered}
B^{-1}\left(B(t)+\frac{1}{n} B(L)\right)<t+\frac{1}{2} L, \\
B(t)+\frac{1}{n} B(L)<B\left(t+\frac{1}{2} L\right), \\
\beta(t)>\frac{1}{n} B(L) .
\end{gathered}
$$

\section{Generation of a Poncelet annulus by an element of the family $\mathcal{B}_{2 \pi, n}$}

We denote by $\mathcal{E}$ a family of all closed strictly convex curves parametrized by support function. The parametric eqnarray of $C \in \mathcal{E}$ is of the form

$$
z(t)=p(t) e^{i t}+\dot{p}(t) i e^{i t} \text { for } t \in[0,2 \pi]
$$

see Santalo (1976) and Bonnesen et al. (1948).

We will assume that $p \in C^{2}$ and

$$
R=p+\ddot{p}>0 .
$$

Theorem 4.1 Each element of the family $\mathcal{B}_{2 \pi, n}$ and a curve $C \in \mathcal{E}$ generates an annulus having a property of the Poncelet porism for a natural number $n$.

Proof Let us fix a natural number $n$ and a function $b \in \mathcal{B}_{2 \pi, n}$. The function $b$ determines a function $\varphi$ given the formula (2.13). We consider a curve $C_{\varphi}$ formed by points of intersection of tangents to $C$ at $z(t)$ and $z(\varphi(t))$. The condition (2.3) implies that the annulus $C C_{\varphi}$ bounded by $C$ and $C_{\varphi}$ is a Poncelet annulus. 


\section{Generation of an element of $\mathcal{B}_{2 \pi, n}$ by a Poncelet annulus}

Theorem 5.1 If $C \in \mathcal{E}$ then for some natural number $n$ each Poncelet annulus $C D$ determines an element of the family $\mathcal{B}_{2 \pi, n}$.

Proof We assume that an annulus $C D$ is a Poncelet one for a natural number $n$ where $C \in \mathcal{E}$ is given by (4.1). Tangent lines to $C$ passing through a point of $D$ intersects $C$ at two points, and these points define a function $\varphi$. Let

$$
b=\left(\varphi^{[n-1]}\right)^{\prime}+\left(\varphi^{[n-2]}\right)^{\prime}+\cdots+\varphi^{\prime}+1 .
$$

Obviously $b \in \mathcal{B}_{2 \pi}$ and $\varphi^{\prime}=\frac{b}{b \circ \varphi}$. We have

$$
B(t)=\varphi^{[n-1]}(t)+\cdots+\varphi(t)+t-\varphi^{[n-1]}(0)-\cdots-\varphi(0) .
$$

We note that

$$
\varphi^{[j]}(2 \pi)=\varphi^{[j]}(0)+2 \pi \text { for all natural } j .
$$

The formulas (5.2) and (5.3) imply

$$
B(2 \pi)=2 \pi n
$$

On the other hand from (5.2) we obtain

$$
\begin{aligned}
B(\varphi(t)) & =\varphi^{[n]}(t)+\varphi^{[n-1]}(t)+\cdots+\varphi(t)-\varphi^{[n-1]}(0)-\cdots-\varphi(0) \\
& =2 \pi+t+\varphi^{[n-1]}(t)+\cdots+\varphi(t)-\varphi^{[n-1]}(0)-\cdots-\varphi(0) \\
& =2 \pi+B(t)
\end{aligned}
$$

i. e.

$$
B \circ \varphi=B+2 \pi .
$$

The inequality $\varphi(t)<t+\pi$ gives us $B(\varphi(t))<B(t+\pi)$. Hence we have

$$
\beta(t)=B(t+\pi)-B(t)>B(\varphi(t))-B(t)=2 \pi=\frac{1}{n} B(2 \pi)
$$

i. e.

$$
\beta_{\min }>\frac{1}{n} B(2 \pi) \quad \text { and } \quad b \in \mathcal{B}_{2 \pi, n}
$$




\section{Circular annuli}

In this section we give the necessary and sufficient condition for a circular annulus to be a Poncelet annulus. We consider two circles $C$ and $D$ defined as follows:

$$
\left\{\begin{array}{l}
C: x^{2}+y^{2}=r^{2} \\
D:(x-a)^{2}+y^{2}=R^{2} \\
0<a<R-r
\end{array}\right.
$$

and the circular annulus $C D$ will be parametrized by

$$
\left\{\begin{array}{l}
C: z(t)=r e^{i t} \\
D: w(t)=z(t)+\lambda(t) i e^{i t} \quad \text { for } t \in[0,2 \pi]
\end{array}\right.
$$

where a function $\lambda$ is given by the formula

$$
\lambda(t)=\sqrt{R^{2}-(r-a \cos t)^{2}}-a \sin t .
$$

Let

$$
\sigma(t)=\frac{1}{R}(r-a \cos t)
$$

We note that

$$
\lambda^{\prime}=\frac{\sigma}{\sqrt{1-\sigma^{2}}} \lambda-r
$$

Theorem 6.1 A circular annulus bounded by the circles $x^{2}+y^{2}=r^{2},(x-a)^{2}+y^{2}=$ $R^{2}$, where $0<a<R-r$ is a Poncelet's annulus if and only if the number

$$
n=\frac{B(2 \pi)}{B\left(2 \arctan \frac{1}{r} \sqrt{R^{2}-(r-a)^{2}}\right)}
$$

is a positive integer, where a function $b$ determining the function $B$ is given by

$$
b(t)=\left[1-\left(\frac{r-a \cos t}{R}\right)^{2}\right]^{\frac{-1}{2}}
$$

and belongs to the class $\mathcal{B}_{2 \pi, n}$.

Proof The tangent line to the circle $C$ at a point $r e^{i t}$ intersects the circle $D$ at a point $w(t)=z(t)+\lambda(t) i e^{i t}$. We pass by $w(t)$ the second tangent line to $C$. It intersects $C$ at a point with a parameter which will be denoted by $\varphi(t)$. It is easy to see that

$$
\tan \frac{\varphi(t)-t}{2}=\frac{\lambda(t)}{r}
$$


Making use of (6.8) and (6.5) we obtain

$$
\varphi^{\prime}=\frac{2 r \sigma \lambda-\left(r^{2}-\lambda^{2}\right) \sqrt{1-\sigma^{2}}}{\left(r^{2}+\lambda^{2}\right) \sqrt{1-\sigma^{2}}} .
$$

The equality (6.4) implies immediately

$$
\begin{aligned}
\sigma \circ \varphi & =\frac{r}{R}-\frac{a}{R} \cos \varphi \\
& =\frac{r}{R}-\frac{a}{R}\left(\frac{r^{2}-\lambda^{2}}{r^{2}+\lambda^{2}} \cos t-\frac{2 r \lambda}{r^{2}+\lambda^{2}} \sin t\right) .
\end{aligned}
$$

Hence we have

$$
\begin{aligned}
R\left(r^{2}+\lambda^{2}\right) \sigma \circ \varphi & =r\left(r^{2}+\lambda^{2}\right)-\left(r^{2}-\lambda^{2}\right) a \cos t+2 r \lambda a \sin t \\
& =r\left(r^{2}+\lambda^{2}\right)-\left(r^{2}-\lambda^{2}\right)(r-R \sigma)+2 r \lambda R \sigma^{\prime} \\
& =2 r \lambda^{2}+2 r \lambda R \sigma^{\prime}+\left(r^{2}-\lambda^{2}\right) R \sigma .
\end{aligned}
$$

We note that

$$
\lambda=R \sqrt{1-\sigma^{2}}-R \sigma^{\prime} .
$$

Thus we have

$$
\begin{aligned}
& \left(r^{2}+\lambda^{2}\right) R \cdot \sigma \circ \varphi \\
& =2 r \lambda\left(\lambda+R \sigma^{\prime}\right)+\left(r^{2}-\lambda^{2}\right) R \sigma \\
& =2 r \lambda R \sqrt{1-\sigma^{2}}+\left(r^{2}-\lambda^{2}\right) R \sigma
\end{aligned}
$$

i.e.

$$
\sigma \circ \varphi=\frac{2 r \lambda \sqrt{1-\sigma^{2}}+\left(r^{2}-\lambda^{2}\right) \sigma}{r^{2}+\lambda^{2}} .
$$

Similarly we have

$$
\begin{aligned}
& \left(r^{2}+\lambda^{2}\right)^{2}\left[1-(\sigma \circ \varphi)^{2}\right] \\
& =\left(r^{2}+\lambda^{2}\right)^{2}\left(1-\left[\frac{2 r \lambda \sqrt{1-\sigma^{2}}+\left(r^{2}-\lambda^{2}\right) \sigma}{r^{2}+\lambda^{2}}\right]^{2}\right) \\
& =r^{4}+2 r^{2} \lambda^{2}+\lambda^{4}-4 r^{2} \lambda^{2}\left(1-\sigma^{2}\right)-4 r \lambda \sqrt{1-\sigma^{2}}\left(r^{2}-\lambda^{2}\right) \sigma-\left(r^{2}-\lambda^{2}\right)^{2} \sigma^{2} \\
& =r^{4}+2 r^{2} \lambda^{2}+\lambda^{4}-4 r^{2} \lambda^{2}+4 r^{2} \lambda^{2} \sigma^{2}-4 r \lambda \sqrt{1-\sigma^{2}}\left(r^{2}-\lambda^{2}\right) \sigma-\left(r^{2}-\lambda^{2}\right)^{2} \sigma^{2} \\
& =\left(r^{2}-\lambda^{2}\right)^{2}-4 r \lambda \sqrt{1-\sigma^{2}}\left(r^{2}-\lambda^{2}\right) \sigma+4 r^{2} \lambda^{2} \sigma^{2}-\left(r^{2}-\lambda^{2}\right)^{2} \sigma^{2}
\end{aligned}
$$




$$
\begin{aligned}
& =\left(1-\sigma^{2}\right)\left(r^{2}-\lambda^{2}\right)^{2}-2 \sqrt{1-\sigma^{2}}\left(r^{2}-\lambda^{2}\right) \cdot 2 r \lambda \sigma+4 r^{2} \lambda^{2} \sigma^{2} \\
& =\left(2 r \lambda \sigma-\left(r^{2}-\lambda^{2}\right) \sqrt{1-\sigma^{2}}\right)^{2}
\end{aligned}
$$

and

$$
\sqrt{1-(\sigma \circ \varphi)^{2}}=\frac{2 r \lambda \sigma-\left(r^{2}-\lambda^{2}\right) \sqrt{1-\sigma^{2}}}{r^{2}+\lambda^{2}}
$$

Comparing (6.9) and (6.12) we get

$$
\varphi^{\prime}=\frac{\sqrt{1-(\sigma \circ \varphi)^{2}}}{\sqrt{1-\sigma^{2}}} .
$$

The coincidence of the value of $\varphi(0)$ given by formula (2.15) for $b$ given by (6.7) and the value of $\varphi(0)$ resulting form the formula (6.8) lead us to the formula (6.6).

Open Access This article is distributed under the terms of the Creative Commons Attribution License which permits any use, distribution, and reproduction in any medium, provided the original author(s) and the source are credited.

\section{References}

Bonnesen, T., Fenchel, W.: Theorie der konvexen Körper. Chelsea Publishing Company, New York (1948) Bos, H.J.M., Kers, C., Dort, F., Raven, D.W.: Poncelet's closure theorem. Expon. Math. 5, 289-364 (1987) Cieślak, W.: Some global properties of plane curves. Publ. Inst. Math. 39(53), 169-172 (1986)

Cieślak, W., Szczygielska, E.: Circuminscribed polygons in a plane annulus. Ann. UMCS, LXII (2008)

Cieślak, W., Martini, H., Mozgawa, W.: On the rotation index of bar billiards and Poncelets porism. Bull. Belgian Math. Soc. Simon Stevin (2013) (to appear)

Mozgawa, W.: Bar billiards and Poncelet's porism. Rend. Sem. Mat. Univ. Padova 120, 157-166 (2008)

Radić, M.: An improved method for establishing Fuss' relations for bicentric polygons. C. R. Math. Acad. Sci. Paris 348, 415-417 (2010)

Radić, M.: Certain relations concerning bicentric polygons and 2-parametric presentation of Fuss' relations. Math. Pannon. 20, 219-248 (2009)

Santalo, L.: Integral geometry and geometric probability. Encyclopedia of mathematics and its applications, Reading (1976)

Weisstein, E. W.: Poncelet's porism, from math world—a wolfram web resource. http://mathworld.wolfram. com/Ponceletsporism.html (2008) 